\title{
Two Long-period Cataclysmic Variable Stars: ASASSN-14ho and V1062 Cyg
}

\author{
L. Claire Gasque ${ }^{1}$, Callum A. Hening ${ }^{1}$, Raphael E. Hviding ${ }^{1,2}{ }^{(1)}$, John R. Thorstensen ${ }^{1}(\mathbb{D})$, Kerry Paterson ${ }^{3}$ (1) \\ Hannes Breytenbach ${ }^{3,4}$, Mokhine Motsoaledi ${ }^{3,4}$, and Patrick A. Woudt ${ }^{3}$ \\ ${ }^{1}$ Department of Physics and Astronomy, Dartmouth College, Hanover, NH 03755, USA \\ ${ }^{2}$ Steward Observatory, University of Arizona, Tucson, AZ 85721, USA \\ ${ }^{3}$ Department Astronomy, University of Cape Town, Private Bag X3, Rondebosch 7701, South Africa \\ ${ }^{4}$ South African Astronomical Observatory, PO Box 9, Observatory 7935, Cape Town, South Africa \\ Received 2019 June 7; revised 2019 August 12; accepted 2019 August 21; published 2019 September 23
}

\begin{abstract}
We report spectroscopy and photometry of the cataclysmic variable stars ASASSN-14ho and V1062 Cyg. Both are dwarf novae with spectra dominated by their secondary stars, which we classify approximately as K4 and M0.5, respectively. Their orbital periods, determined mostly from the secondary stars' radial velocities, proved to be nearly identical, respectively $350.14 \pm 0.15$ and $348.25 \pm 0.60$ minutes. The $\mathrm{H} \alpha$ emission line in V1062 Cyg displays a relatively sharp emission component that tracks the secondary's motion, which may arise on the irradiated face of the secondary; this is not often seen and may indicate an unusually strong flux of ionizing radiation. Both systems exhibit double-peaked orbital modulation consistent with ellipsoidal variation from the changing aspect of the secondary. We model these variations to constrain the orbital inclination $i$, and estimate approximate component masses based on $i$ and the secondary velocity amplitude $K_{2}$.

Unified Astronomy Thesaurus concepts: Cataclysmic variable stars (203); Dwarf novae (418); Close binary stars (254); Ellipsoidal variable stars (455)
\end{abstract}

\section{Introduction}

Cataclysmic variable stars (CVs) are binary stars in which a white dwarf primary accretes matter from a more extended secondary star via Roche-lobe overflow. The accreting matter usually forms a disk around the primary. A good overview of CVs is presented in Warner (1995).

Dwarf novae (DN), one of the most common types of CV, are weakly or non-magnetic systems which can spontaneously go from quiescence into outburst, a more luminous state corresponding to a brightening of several magnitudes. DN outbursts are thought to be caused by the rapid release of gravitational potential energy triggered by instabilities in the accretion disk. At longer orbital periods, the quiescent spectra of DN are often dominated by the spectrum of a late-type secondary (Warner 1995).

DN systems in which the secondary star is the dominant flux contributor often show a modulation in the light curve caused by the tidal distortions in the secondary star (Barwig \& Schoembs 1983). The projection effects cause two maxima per orbital period at the quadrature phases, and gravity darkening causes the side of the secondary facing the white dwarf to appear darker than the back side, resulting in unequal minima (Bochkarev et al. 1979).

This work focuses on two DN systems: ASASSN-14ho and V1062 Cyg. We obtained optical spectroscopy and photometry of these systems in order to determine their orbital periods and estimate the spectral types of their secondary stars and the binary system parameters.

ASSASN-14ho $\quad$ (R.A. $=06^{\mathrm{h}} 30^{\mathrm{m}} 27^{\mathrm{s}} 3, \quad$ decl. $=-65^{\circ} 29^{\prime \prime}$ 50 '2, J2000) was announced as a CV in outburst following observations taken of the system on the du Pont $2.5 \mathrm{~m}$ spectrograph at the Las Campanas Observatory in 2014 September (Prieto et al. 2014). The system had a quiescent brightness of $V=15.4$ on 2014 September 9 and reached $V=10.92$ on 2014 September 11 before declining. A previous outburst had been seen in 2009 by the Catalina Real Time
Transient Survey (Drake et al. 2009). Inverting the Gaia Data Release 2 (DR2) parallax (Gaia Collaboration et al. 2016, 2018) gives a distance estimate of $331 \pm 3 \mathrm{pc}$.

V1062 Cyg (R.A. $=21^{\mathrm{h}} 08^{\mathrm{m}} 12^{\mathrm{s}} \cdot 20^{\mathrm{s}}$, decl. $=+36^{\circ} 49^{\prime \prime} 27 ! 7$, J2000) was first reported by Hoffmeister (1965), with a magnitude range of $15.5-18 \mathrm{mag}$. Despite having since appeared in several CV catalogs, the system has apparently never been studied in detail and its orbital period remained unknown. The inverse of its Gaia DR2 parallax is 840 $(+95,-78)$ pc.

We describe our observations, instruments, and analysis in Section 2. In Sections 3 and 4 we present our results for ASSASN-14ho and V1062 Cyg, respectively. Finally, our conclusions and discussion are presented in Section 5.

\section{Methods \\ 2.1. Observations}

Our observations of ASASSN-14ho were taken in 2017 February at the South African Astronomical Observatory (SAAO) near Sutherland. Observations of V1062 Cyg were taken in 2017 June at the MDM Observatory on Kitt Peak, Arizona.

Our spectra of ASASSN-14ho were taken with the Spectrograph Upgrade-Newly Improved Cassegrain (SpUpNIC) instrument (Crause et al. 2016) mounted on the SAAO $1.9 \mathrm{~m}$ telescope. We used the G6 grating (blazed for $4600 \AA$ ) which gave a dispersion of $1.36 \mathrm{Apx}^{-1}$. A 1 !' 35 slit and a grating angle of 11.75 yielded a resolving power of 1000 from 4200 to $7000 \AA$. We obtained 43 spectra of ASASSN-14ho, mostly $600 \mathrm{~s}$ exposures, over five days, for a total integration time of just over $7.5 \mathrm{hr}$. Table 1 gives the times of observation and radial velocities derived from the spectra (described in Section 2.3). 
Table 1

Radial Velocities of ASASSN-14ho

\begin{tabular}{|c|c|c|}
\hline Time $^{\mathrm{a}}$ & $\begin{array}{c}v_{\text {abs }} \\
\left(\mathrm{km} \mathrm{s}^{-1}\right)\end{array}$ & $\frac{v_{\mathrm{emn}}}{\left(\mathrm{km} \mathrm{s}^{-1}\right)^{\mathrm{b}}}$ \\
\hline 57794.3628 & $318 \pm 10$ & $-45 \pm 13$ \\
\hline 57794.3698 & $319 \pm 11$ & $-35 \pm 14$ \\
\hline 57794.3771 & $269 \pm 12$ & $-55 \pm 16$ \\
\hline 57794.3841 & $268 \pm 10$ & $-39 \pm 15$ \\
\hline 57794.3984 & $201 \pm 11$ & $-62 \pm 14$ \\
\hline 57794.4054 & $154 \pm 11$ & $-43 \pm 16$ \\
\hline 57794.4124 & $128 \pm 15$ & $-6 \pm 16$ \\
\hline 57794.4193 & $63 \pm 13$ & $-7 \pm 21$ \\
\hline 57794.4287 & $41 \pm 30$ & $56 \pm 20$ \\
\hline 57794.4357 & $-38 \pm 15$ & $80 \pm 22$ \\
\hline 57794.4427 & $-45 \pm 12$ & $72 \pm 18$ \\
\hline 57794.4497 & $-98 \pm 12$ & $63 \pm 23$ \\
\hline 57794.4594 & $-131 \pm 13$ & $11 \pm 26$ \\
\hline 57794.4664 & $-143 \pm 11$ & $66 \pm 21$ \\
\hline 57794.4734 & $-152 \pm 12$ & $90 \pm 23$ \\
\hline 57794.4804 & $-173 \pm 10$ & $76 \pm 27$ \\
\hline 57794.4895 & $-148 \pm 13$ & $123 \pm 20$ \\
\hline 57794.4964 & $-145 \pm 12$ & $119 \pm 21$ \\
\hline 57794.5034 & $-140 \pm 11$ & $70 \pm 18$ \\
\hline 57794.5104 & $-106 \pm 28$ & $108 \pm 16$ \\
\hline 57794.5205 & $-67 \pm 11$ & $101 \pm 17$ \\
\hline 57794.5275 & $-20 \pm 19$ & $100 \pm 19$ \\
\hline 57794.5345 & $18 \pm 12$ & $57 \pm 25$ \\
\hline 57794.5415 & $75 \pm 14$ & $\cdots$ \\
\hline 57794.5498 & $125 \pm 13$ & $\cdots$ \\
\hline 57794.5572 & $112 \pm 34$ & $62 \pm 26$ \\
\hline 57794.5642 & $208 \pm 15$ & $\ldots$ \\
\hline 57794.5712 & $237 \pm 19$ & $\ldots$ \\
\hline 57796.2883 & $300 \pm 11$ & $-34 \pm 16$ \\
\hline 57796.2953 & $311 \pm 9$ & $-26 \pm 16$ \\
\hline 57796.3023 & $302 \pm 9$ & $-78 \pm 16$ \\
\hline 57796.3093 & $316 \pm 10$ & $-46 \pm 17$ \\
\hline 57796.3173 & $290 \pm 9$ & $-43 \pm 21$ \\
\hline 57796.3243 & $271 \pm 10$ & $-24 \pm 19$ \\
\hline 57796.3313 & $246 \pm 10$ & $-40 \pm 20$ \\
\hline 57796.3383 & $218 \pm 10$ & $-72 \pm 21$ \\
\hline 57796.3500 & $164 \pm 11$ & $-59 \pm 24$ \\
\hline 57796.3570 & $104 \pm 9$ & $-37 \pm 21$ \\
\hline 57797.2745 & $295 \pm 10$ & $-31 \pm 20$ \\
\hline 57797.2862 & $267 \pm 10$ & $-33 \pm 17$ \\
\hline 57797.2978 & $254 \pm 8$ & $-36 \pm 18$ \\
\hline 57799.2718 & $150 \pm 8$ & $\ldots$ \\
\hline 57799.2802 & $94 \pm 8$ & $\cdots$ \\
\hline
\end{tabular}

Notes.

${ }^{a}$ Barycentric Julian date of mid-integration, minus 2,400,000, in the UTC time system.

${ }^{\mathrm{b}}$ Computed using a convolution function with positive and negative Gaussians separated by $44 \AA$, each with a FWHM of $7 \AA$.

The V1062 Cyg spectra were taken with the modspec ${ }^{5}$ spectrograph on the $2.4 \mathrm{~m}$ Hiltner telescope. We used a $600 \mathrm{~mm}^{-1} 5000 \AA$ blazed grating and a $2048 \times 2048$ 24 micron pixel Scientific Imaging Technologies, Inc (SITe) CCD, which gave a wavelength range of $4300-7500 \AA$ and a resolution of $3.4 \AA$. Table 2 gives the times of observation and radial velocities derived from the spectra.

The photometry of ASASSN-14ho is from the Sutherland High Speed Optical Camera (SHOC; Coppejans et al. 2013) on

\footnotetext{
5 http://mdm.kpno.noao.edu/Manuals/ModSpec/modspec_man.html
}

Table 2

Radial Velocities of V1062 Cyg

\begin{tabular}{|c|c|c|c|}
\hline $\begin{array}{l}\text { Time }^{\mathrm{a}} \\
\left(\mathrm{km} \mathrm{s}^{-1}\right)\end{array}$ & $\begin{array}{c}v_{\mathrm{abs}} \\
\left(\mathrm{km} \mathrm{s}^{-1}\right)\end{array}$ & $\begin{array}{c}\mathrm{H} \alpha \text { wing }^{\mathrm{b}} \\
\left(\mathrm{km} \mathrm{s}^{-1}\right)\end{array}$ & $\mathrm{H} \alpha$ peak $^{\mathrm{c}}$ \\
\hline 57928.7553 & $139 \pm 48$ & $\ldots$ & $112 \pm 34$ \\
\hline 57928.7640 & $83 \pm 33$ & $\cdots$ & $92 \pm 23$ \\
\hline 57928.7727 & $171 \pm 30$ & $\cdots$ & $49 \pm 22$ \\
\hline 57928.7814 & $158 \pm 32$ & $\ldots$ & $94 \pm 20$ \\
\hline 57928.7931 & $110 \pm 31$ & $-185 \pm 39$ & $57 \pm 22$ \\
\hline 57928.8074 & $146 \pm 26$ & $-202 \pm 27$ & $81 \pm 15$ \\
\hline 57928.8602 & $-107 \pm 30$ & $-45 \pm 31$ & $-60 \pm 23$ \\
\hline 57928.8745 & $-163 \pm 29$ & $12 \pm 32$ & $-76 \pm 22$ \\
\hline 57928.8888 & $-207 \pm 25$ & $-36 \pm 25$ & $-147 \pm 16$ \\
\hline 57928.9031 & $-227 \pm 23$ & $-7 \pm 30$ & $-157 \pm 17$ \\
\hline 57928.9174 & $-213 \pm 23$ & $18 \pm 31$ & $-153 \pm 18$ \\
\hline 57928.9316 & $-204 \pm 29$ & $72 \pm 36$ & $-66 \pm 20$ \\
\hline 57928.9459 & $-99 \pm 18$ & $3 \pm 28$ & $-85 \pm 15$ \\
\hline 57928.9602 & $-33 \pm 25$ & $-43 \pm 33$ & $-90 \pm 21$ \\
\hline 57929.8507 & $-160 \pm 28$ & $34 \pm 38$ & $-221 \pm 19$ \\
\hline 57929.8684 & $-215 \pm 22$ & $81 \pm 24$ & $-143 \pm 14$ \\
\hline 57929.8862 & $\cdots$ & $\cdots$ & $-80 \pm 31$ \\
\hline 57930.7428 & $\cdots$ & $-147 \pm 30$ & $\cdots$ \\
\hline 57930.7605 & $72 \pm 35$ & $-186 \pm 25$ & $31 \pm 30$ \\
\hline 57930.7783 & $-81 \pm 44$ & $\cdots$ & $\cdots$ \\
\hline 57930.7960 & $-153 \pm 46$ & $-75 \pm 34$ & $-172 \pm 33$ \\
\hline 57930.8137 & $-189 \pm 29$ & $-41 \pm 30$ & $-224 \pm 20$ \\
\hline 57930.8331 & $-196 \pm 33$ & $67 \pm 25$ & $-184 \pm 21$ \\
\hline 57930.9336 & $93 \pm 27$ & $-198 \pm 19$ & $68 \pm 23$ \\
\hline
\end{tabular}

Notes.

${ }^{a}$ Barycentric Julian date of mid-integration, minus 2,400,000, in the UTC time system.

${ }^{\mathrm{b}}$ Radial velocity of the $\mathrm{H} \alpha$ line wings, measured using a convolution function consisting of positive and negative Gaussians with FWHM $10 \AA$ A separated by $32 \AA$.

${ }^{c}$ Radial velocity of the $\mathrm{H} \alpha$ line peak, measured using a convolution function formed from the derivative of a Gaussian, optimized for a line with FWHM $12 \AA$.

Table 3

Photometric Observations of ASASSN-14ho

\begin{tabular}{|c|c|c|c|c|c|}
\hline \multirow{2}{*}{ Date } & \multirow{2}{*}{$\begin{array}{l}\text { Time } \\
\text { (UTC) }\end{array}$} & \multirow{2}{*}{$\begin{array}{l}\text { Exp } \\
\text { (s) }\end{array}$} & \multirow{2}{*}{$N$} & \multicolumn{2}{|c|}{ Airmass } \\
\hline & & & & Start & End \\
\hline 2017 Feb 11 & $18: 44: 21$ & 10.0 & 450 & 1.2079 & 1.1954 \\
\hline 2017 Feb 11 & 19:59:41 & 15.0 & 320 & 1.1954 & 1.24 \\
\hline 2017 Feb 11 & $21: 20: 10$ & 25.0 & 355 & 1.2429 & 1.5361 \\
\hline
\end{tabular}

the SAAO $1.0 \mathrm{~m}$ telescope. The instrument uses a backilluminated frame transfer CCD with a $6.76 \mathrm{~ms}$ dead time. The system offers a $2 ! 85 \times 2 ! .85$ field of view with a plate scale of 0 ". $668 \mathrm{px}^{-1}$ in the $4 \times 4$ binning mode. Our observations, all from 2017 February 11, consist of 1125 unfiltered frames obtained over five hours. Table 3 gives the times of observations, length of exposure, number of frames taken, and airmass.

Our photometry of V1062 Cyg was taken with an Andor Ikon DU-937N camera on the MDM $1.3 \mathrm{~m} \mathrm{McGraw-Hill}$ Telescope. The camera has $512 \times 51213 \mu \mathrm{m}^{2}$ pixels which gave a $2 ! 32 \times 2 ! 32$ field of view (Thorstensen 2013). Over the course of two nights, 720 frames were taken for a total integration time of twelve hours. Table 4 gives the times of observations, length of exposure, number of frames taken, and airmass. 
Table 4

Photometric Observations of V1062 Cyg

\begin{tabular}{|c|c|c|c|c|c|}
\hline \multirow{2}{*}{ Date } & \multirow{2}{*}{$\begin{array}{c}\text { Time } \\
\text { (UTC) }\end{array}$} & \multirow{2}{*}{$\begin{array}{l}\text { Exp } \\
\text { (s) }\end{array}$} & \multirow{2}{*}{$N$} & \multicolumn{2}{|c|}{ Airmass } \\
\hline & & & & Start & End \\
\hline 2017 Jun 27 & $05: 15: 57$ & 60.0 & 360 & 1.991 & 1.030 \\
\hline 2017 Jun 28 & 05:03:12 & 60.0 & 360 & 2.094 & 1.023 \\
\hline
\end{tabular}

\subsection{Data Reduction}

We reduced our spectra using procedures broadly similar to those outlined in Thorstensen et al. (2016). We used IRAF ${ }^{6}$ for bias and flat-field corrections. For the SAAO spectra we extracted background-subtracted one-dimensional spectra using the IRAF apall task, and applied wavelength calibrations derived from $\mathrm{Cu}-\mathrm{Ar}$ arc spectra interleaved with the stellar exposures. For the MDM data, we extracted the spectra using a local implementation of the algorithm given by Horne (1986), and a wavelength scale derived by rigidly shifting a master pixel-to-wavelength solution using the $\lambda 5577$ airglow line as a fiducial. At both observatories we derived a flux calibration using observations of standard stars taken during the run.

Both the SAAO SHOC and the MDM Andor camera write three-dimensional data cubes. To process these we used python scripts to (1) create average bias images and flat-field frames from exposures of the twilight sky, (2) subtract the bias and divide by the flat field, and (3) derive barycentric times of the exposure centers. We then split the individual frames from the data cube and measured the magnitudes of the program object and several comparison stars using the aperture photometry task in the IRAF implementation of DAOPHOT (Stetson 1987), and finally compiled the differential magnitudes into a time series. The uncertainties in our program star measurements were estimated from the scatter in the differential magnitudes of apparently constant stars of similar brightness.

Our data cannot be transformed accurately to a standard passband because the SAAO data were taken unfiltered, and the filter used at MDM was intended only to mitigate scattered moonlight (passing $\lambda>4200 \AA$ ). However, we did transform to approximate $V$ magnitude by adding the $V$ magnitudes of our comparison stars to the differential magnitudes. These pseudo$V$ magnitudes should not be very far off, since in both systems the late-type secondary star dominates the light; the color mismatch between the $\mathrm{CV}$ and the comparison star is therefore unlikely to be severe.

\subsection{Analysis}

We measured radial velocities of the secondary stars using the cross-correlation technique of Tonry \& Davis (1979). For the template spectrum, we used the average of 76 spectra of Gand K-type stars, taken with the Hiltner telescope and modspec in the same configuration used here for V1062 Cyg, that had been shifted to zero apparent velocity prior to averaging.

To measure emission-line velocities, we used a convolution technique developed by Schneider \& Young (1980), in which the line profile is convolved with an odd function and the zero of the convolution is taken as a measure of the line center. For

\footnotetext{
6 IRAF is distributed by the National Optical Astronomy Observatory, which is operated by the Association of Universities for Research in Astronomy (AURA), Inc., under cooperative agreement with the National Science Foundation.
}

the odd function, we used either the derivative of a Gaussian (dgau for short) or the sum of positive and negative Gaussians, offset by a selectable width (gau2). In both cases, only the $\mathrm{H} \alpha$ line gave usable velocities.

To search for periods, we fit general least-squares sinusoids to the radial velocities at each frequency in a dense grid of trial frequencies. In both stars studied here, the adopted orbital frequency had by far the lowest scatter around the best fit, and there was no ambiguity in the daily cycle count. We fit the radial velocities with functions of the form

$$
v(t)=K \sin \left(\frac{2 \pi\left(t-T_{0}\right)}{P}\right)+\gamma
$$

where $P$ is the orbital period and $T_{0}$ is an epoch of blue-to-red crossing chosen to be near the center of the time series. If the velocities faithfully trace the motion of one component, then $\gamma$ will be the systemic radial velocity and $K$ will be the line-ofsight component of that star's orbital velocity. We used a Monte Carlo simulation to estimate the uncertainties in the parameters of our best-fitting sinusoid.

To estimate the secondary stars' spectral types, we began by shifting our individual flux-calibrated spectra to the secondary's rest frame and averaging them. We then subtracted spectra of known spectral type taken from the same instrument, varying the spectral type and normalization with the aim of retrieving a smooth continuum as judged by eye. This resulted in a range of possible classifications and $V$-band magnitudes for the secondary.

To display the spectrum as a function of orbital phase, we set up a grid of 100 evenly spaced fiducial phases around the orbital cycle, and computed average spectra for each phase. Weights for the spectra near each fiducial phase were computed using a Gaussian weighting function, with a standard deviation of 0.03 cycles. The spectra were rectified (divided by a continuum fit) and cleaned of obvious artifacts before being averaged. We then stacked the phase averages into a twodimensional image, and generated gray scale figures from these.

The light curves of both objects showed two peaks per orbit and unequal minima, as expected from the changing aspect of a tidally distorted secondary star. To explore this, we used a modeling program described by Thorstensen \& Armstrong (2005). The program models the Roche-lobe-filling secondary star as a 500-sided polyhedron and computes the $V$-band light curve for the source as observed from Earth. The model parameters are the (assumed known) orbital period, the masses of both stars, the orbital inclination $i$, the secondary's effective temperature $T_{\text {eff }}$, limb- and gravity-darkening coefficients, the distance and reddening of the system, and an assumed flat (in flux per unit wavelength $F_{\lambda}$ ) extra light contribution from the primary and accretion structures. Our estimates of $T_{\text {eff }}$ and the extra light are guided by the spectral decompositions used to estimate the secondary's spectral type. For all calculations we used a linear limb-darkening coefficient of 0.78 , from the tabulations of Claret et al. (2012) for $T_{\text {eff }}=4500 \mathrm{~K}$ and the Kepler passband, which is similar to our observed bands.

From $P$ and the secondary star's velocity semi-amplitude $K_{2}$, we compute the mass function

$$
f=\frac{\left(M_{1}\right)^{3}}{\left(M_{1}+M_{2}\right)^{2}} \sin ^{3}(i)=\frac{P}{2 \pi G}\left(K_{2}\right)^{3} .
$$


Table 5

Fits to Radial Velocities

\begin{tabular}{|c|c|c|c|c|c|c|}
\hline Data set & $T_{0}{ }^{\mathrm{a}}$ & $\begin{array}{c}P \\
\text { (days) }\end{array}$ & $\begin{array}{c}K \\
\left(\mathrm{~km} \mathrm{~s}^{-1}\right)\end{array}$ & $\begin{array}{c}\gamma \\
\left(\mathrm{km} \mathrm{s}^{-1}\right)\end{array}$ & $N$ & $\begin{array}{c}\mathrm{rms} \\
\left(\mathrm{km} \mathrm{s}^{-1}\right)\end{array}$ \\
\hline ASASSN-14ho absorption & $2457794.5417(10)$ & $0.24316(10)$ & $236(5)$ & $69(4)$ & 43 & 13 \\
\hline ASASSN-14ho emission & $2457794.436(7)$ & {$[0.24316]^{\mathrm{b}}$} & $78(10)$ & $29(8)$ & 37 & 22 \\
\hline V1062 Cyg absorption & $2457929.931(2)^{\mathrm{c}}$ & $0.2418(5)^{\mathrm{c}}$ & $184(7)$ & $-32(5)$ & 22 & 24 \\
\hline V1062 Cyg H $\alpha$ cores & $57929.927(9)$ & $0.2418^{\mathrm{b}}$ & $126(20)$ & $-37(17)$ & 23 & 41 \\
\hline V1062 Cyg H $\alpha$ wings & $57929.818(7)$ & $0.2418^{\mathrm{b}}$ & $129(19)$ & $-88(15)$ & 18 & 38 \\
\hline
\end{tabular}

Notes.

a Barycentric Julian date of mid-integration minus 2,440,000. The time system is UTC.

${ }^{b}$ Period fixed to the value derived from the absorption-line fit.

${ }^{\mathrm{c}}$ Light curves taken on subsequent nights suggest a slightly earlier $T_{0}$ and a slightly shorter $P$ than the best fit to the velocities (Section 4.3 ).

We use this to constrain the secondary star's mass $M_{2}$ by estimating a range of plausible inclinations $i$ from the lightcurve model fits, and assuming that the white dwarf mass $M_{1}$ lies in a reasonable range (Ritter \& Burkert 1986; Kepler et al. 2007; Schreiber et al. 2016). Sections 3.3 and 4.3 detail this process for for ASASSN-14ho and V1062 Cyg respectively.

\section{ASASSN-14ho Results}

\subsection{Period and Radial Velocities}

The absorption-line radial velocities are modulated with an unambiguous period of $350.14 \pm 0.15$ minutes, which we adopt as $P_{\text {orb }}$. The $\mathrm{H} \alpha$ line is double-peaked with a FWHM of $\sim 31 \AA$. We measured its radial velocity using a gau2 convolution function with a separation of $44 \mathrm{~A}$, which emphasized the steep sides and wings of the line. The emission velocities showed modulation at essentially the same period as the absorption, but with greater scatter. Table 5 gives parameters of sinusoidal fits to the emission and absorption velocities, and Figure 1 shows the velocities plotted as a function of phase, along with the best-fit sinusoids.

The systemic radial velocity $\gamma$ derived from the absorption lines differs by $42 \mathrm{~km} \mathrm{~s}^{-1}$ from the value from the emission lines. We do not think this discrepancy is significant, because the emission-line velocities do not necessarily trace the white dwarf motion (North et al. 2002). If the emission originated directly from the white dwarf, we would expect an emissionabsorption phase offset of 0.5 cycle. Our fitted sinusoids show a difference of $0.56 \pm 0.03$ cycles, suggesting that the emission from the accretion disk does not track the white dwarf's position exactly. The emission velocities are uncertain enough that we are reluctant to use them to compute a mass ratio.

Figure 2 shows a single-trailed plot of the spectral features of ASASSN-14ho, centered on the $\mathrm{H} \alpha$ emission line. The $\mathrm{H} \alpha$ profile is complex, but has the distinct double-peaked structure characteristic of an accretion disk.

\subsection{Spectral Type}

We find the secondary's spectral type to be near K4V, with an estimated uncertainty of \pm 2 subclasses. The secondary star contributes most of the light in the visible (Figure 3). The subtracted spectrum is characterized by a fairly flat continuum with broad hydrogen emission lines, typical of DN at minimum light (Warner 1995).

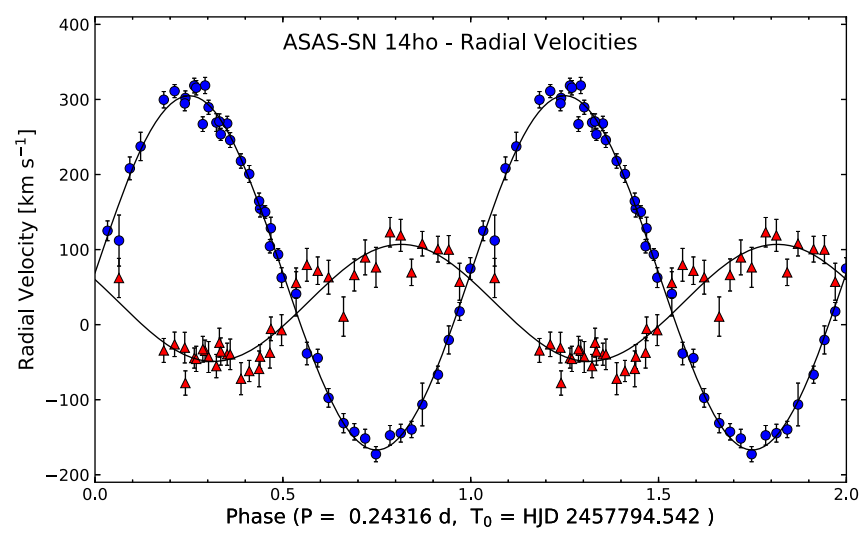

Figure 1. ASASSN-14ho radial velocities folded on the orbital period, with one cycle repeated once for clarity. The blue circles are absorption-line velocities of the secondary star, and the red triangles are velocities of the $\mathrm{H} \alpha$ emission-line wings.

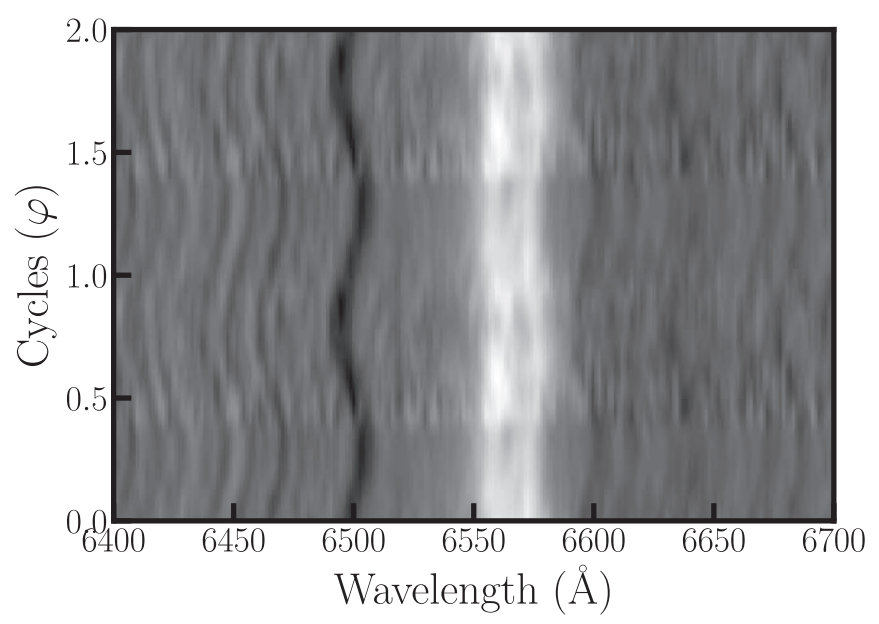

Figure 2. Single-trailed spectrum on ASASSN-14ho, centered on the $\mathrm{H} \alpha$ emission line.

\subsection{Photometry and Stellar Parameters}

Figure 4 shows our photometry folded on the period and phase determined in Section 3.1. As noted earlier, we transformed our instrumental magnitudes to rough apparent $V$ magnitudes using $V=15.25$ for the comparison star, ${ }^{7}$ taken from the AAVSO Photometric All-Sky Survey (APASS) catalog (Henden et al. 2015). The light curve shows an

$\alpha=6: 30: 24.87, \delta=-65: 31: 21.3$. 


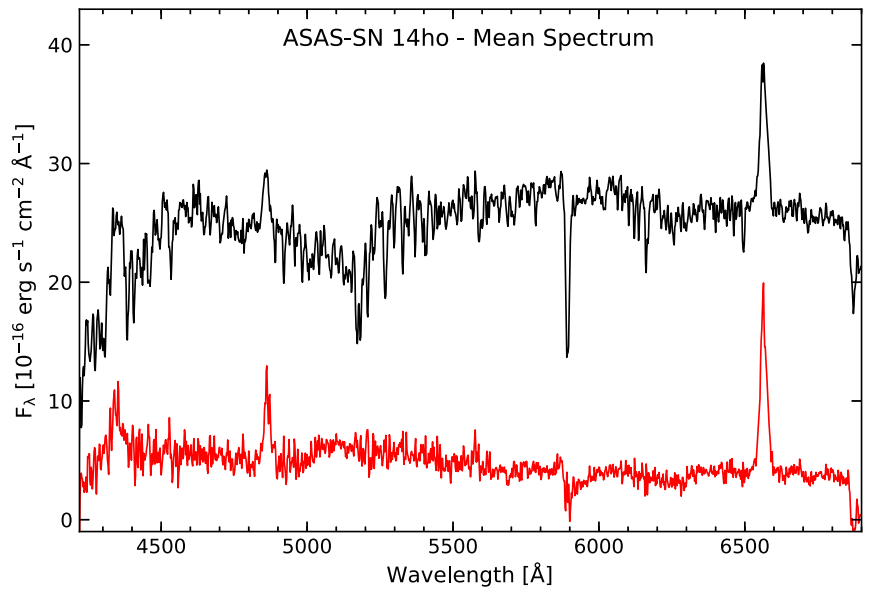

Figure 3. Average spectrum of ASASSN-14ho (black) together with the the spectrum after subtraction (red) of a scaled spectrum of the K4V star Gliese 570a. The individual spectra of ASASSN-14ho were shifted to the rest frame of the secondary star before they were summed.

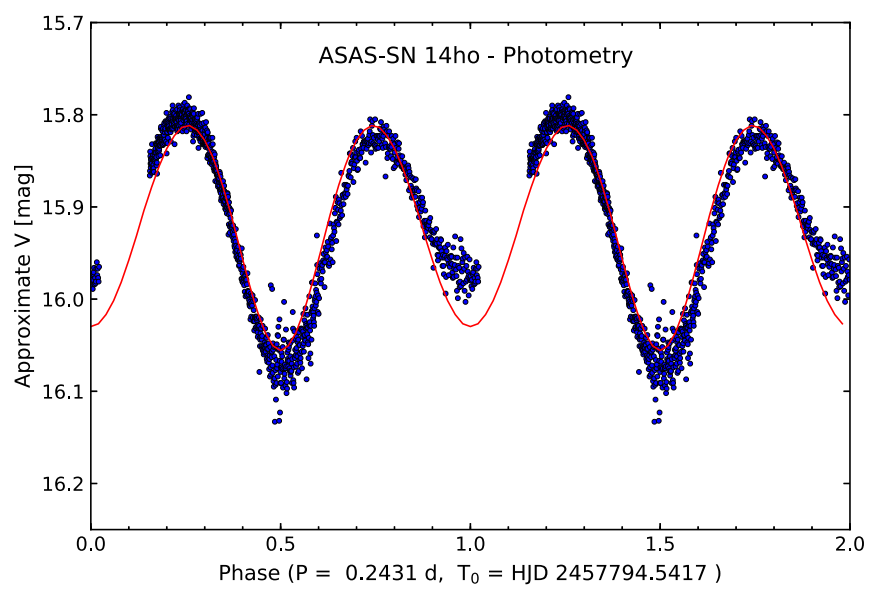

Figure 4. Differential magnitudes of ASASSN-14ho transformed to approximate $V$ plotted as a function of orbital phase, repeated once for clarity. The red curve shows a model of the ellipsoidal variation.

obvious modulation with two peaks per orbit and unequal minima, as expected from a tidally distorted secondary (socalled ellipsoidal variation), and no evidence of an eclipse. The red curve in Figure 4 shows a light curve generated by the light-curve modeling program described earlier. We could not fit all the features in the light curve, such as the extra brightness near phase zero, with perfect fidelity. Therefore, we did not attempt a formal best fit, but adjusted the fit parameters by hand. The fit shown has $M_{1}=1.00$ and $M_{2}=0.28 M_{\odot}$, $i=52^{\circ}$, a secondary effective temperature of $4200 \mathrm{~K}$, and extra light equivalent to $F_{\lambda}=5 \times 10^{-16} \mathrm{erg} \mathrm{s}^{-1} \mathrm{~cm}^{-2} \AA^{-1}$, which gives a reddening-corrected distance nearly identical to the inverse of the Gaia DR2 parallax, and matches the observed mass function. The amplitude of the modeled curve is influenced mostly by the inclination, the estimated contribution from the disk, constant extra light term, and the gravitydarkening coefficient. With the last two factors held constant, we found reasonable fits in the range of $i=52^{\circ} \pm 5^{\circ}$. Varying $M_{2}$ mostly affects the normalization, because the Roche-lobe radius scales roughly as $M_{2}^{1 / 3}$.

In Figure 5, we show the constraints on $M_{1}$ and $M_{2}$ set by the velocity amplitude, for several inclination angles consistent with the light curve. The masses are sensitive to small changes

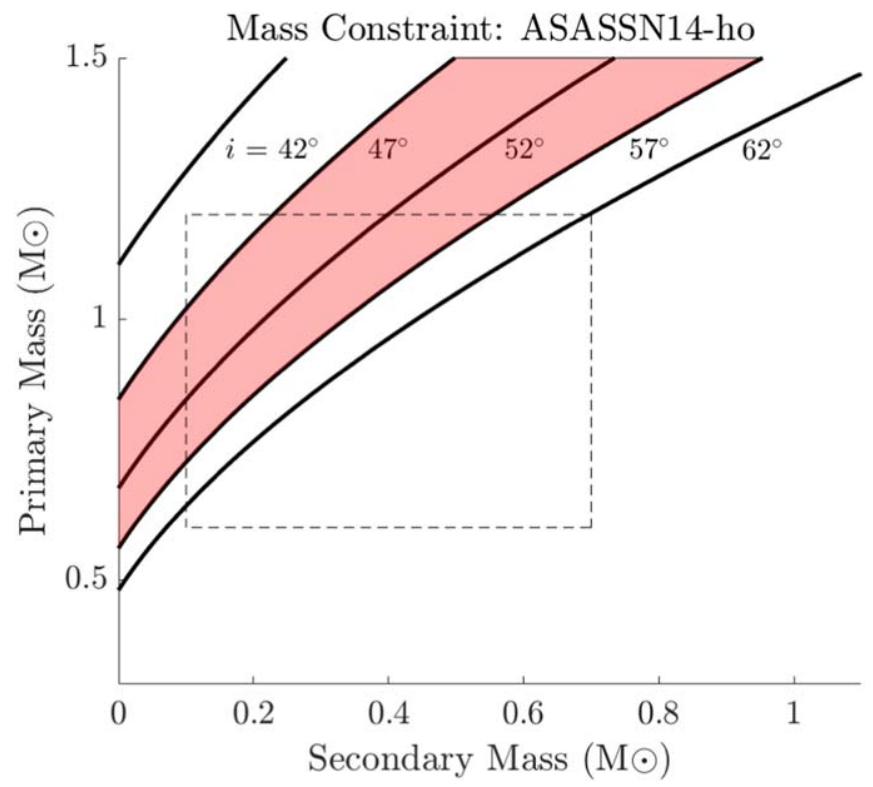

Figure 5. Constraining masses of ASASSN-14ho using the mass function. The shaded area represents the inclination range we report as most reasonable based on the photometry modeling. The dotted box represents a reasonable range of masses for each component in the system.

in inclination. The box in the figure represents a rough range of reasonable masses for both components. If our inclination estimate is correct, the white dwarf mass is likely to be greater than $\sim 0.8$ solar masses and the secondary mass is likely to be rather low.

\section{V1062 Cyg Results}

\subsection{Period and Radial Velocities}

The absorption-line velocities in V1062 Cyg give an unambiguous $P_{\text {orb }}=348.25 \pm 0.84$ minutes (note that the photometry discussed in 4.3 suggests a slightly shorter period). The emission-line velocities corroborate the period found in the absorption lines. The $\mathrm{H} \alpha$ emission line showed a complex structure, with the wings and core of the line moving roughly in antiphase. To measure the line wing velocities we used a gau2 function separated by $32 \AA$, and for the core we used a dgau optimized for $12 \AA$ FWHM. Table 5 gives parameters of leastsquares sinusoidal fits, and Figure 6 plots the velocities of the absorption lines and the two components of the $\mathrm{H} \alpha$ line as a function of orbital phase, together with the best fits.

Figure 7 shows a phase-resolved gray scale representation of the spectrum centered on the $\mathrm{H} \alpha$ emission-line. The velocity modulation of the bright line core is nearly in phase with the absorption, while the weaker, more diffuse wing component appears to move in antiphase. This suggests that the core emission arises from the secondary star, caused perhaps from irradiation of the side facing the white dwarf (Steeghs et al. 2001), or possibly from magnetic activity. The velocity modulation of the wings is apparently consistent with the accretion disk.

\subsection{Spectral Type}

The spectral type of the secondary is near M0.5. The total and subtracted spectra of V1062 Cyg are presented together in Figure 8. 


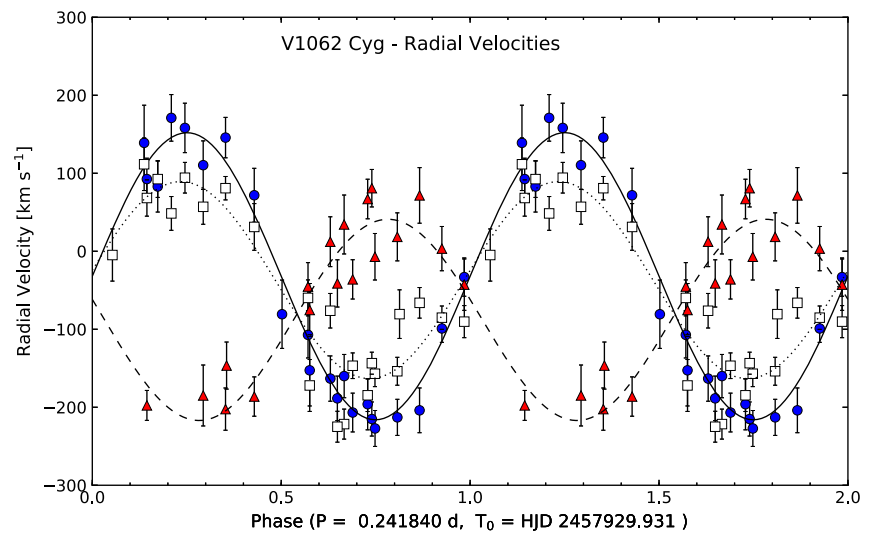

Figure 6. Radial velocities from V1062 Cyg folded on the adopted orbital period and repeated once for clarity, together with the best-fitting sinusoids at the adopted orbital period. The symbols are as follows: blue circles and solid line: absorption-line velocities of the secondary; red triangles and dashed curve: $\mathrm{H} \alpha$ emission-line wings; open squares and dotted curve: $\mathrm{H} \alpha$ emissionline core.

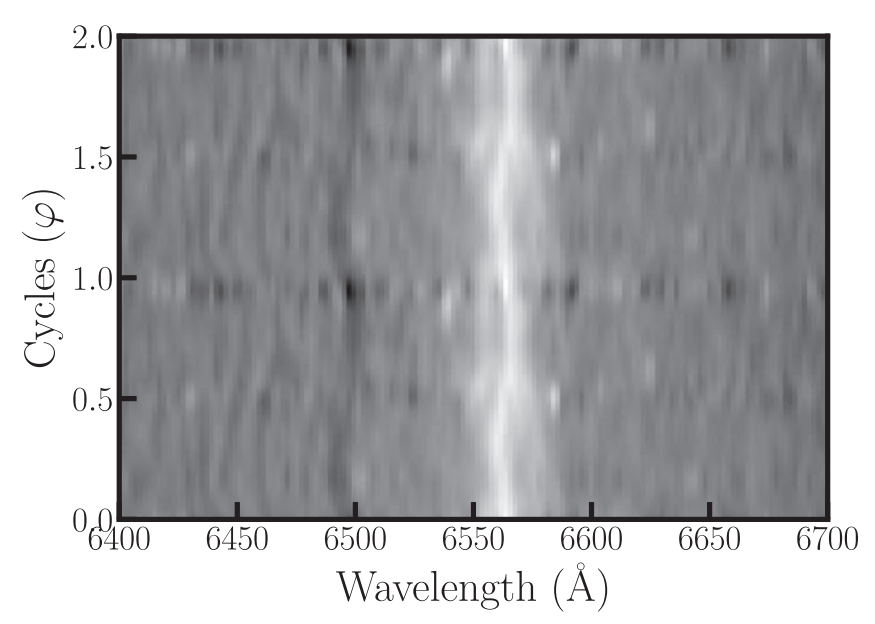

Figure 7. Single-trailed spectrum on V1062 Cyg, centered on the $\mathrm{Ha}$ emission line.

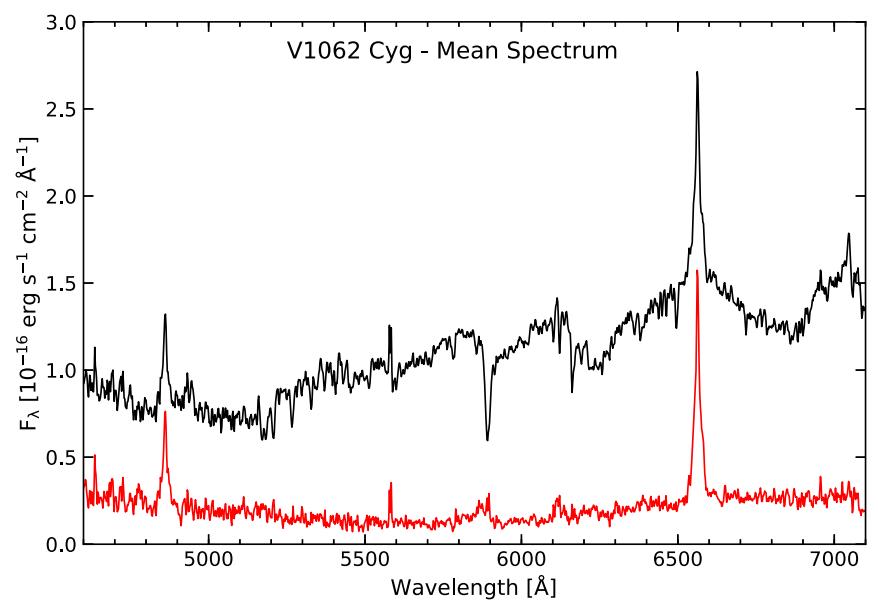

Figure 8. Average spectrum of V1062 Cyg; the lower trace (red) shows the spectrum after subtraction of a scaled spectrum of Gliese 486 (type M0.5V).

\subsection{Photometry and Stellar Parameters}

Figure 9 shows the light curve, which displays a doublepeaked structure similar to ASSASN-14ho (Section 3.3). The ellipsoidal model does not fit as well as for ASASSN-14ho, but

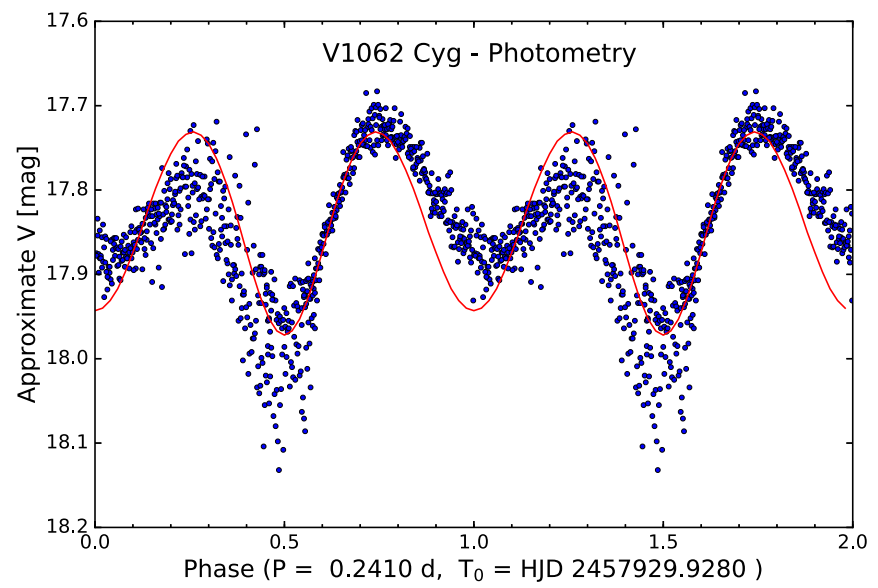

Figure 9. Differential magnitudes of V1062 Cyg transformed to approximate $V$ plotted as a function of orbital phase, repeated once for clarity. The red curve shows a model of the ellipsoidal variation.

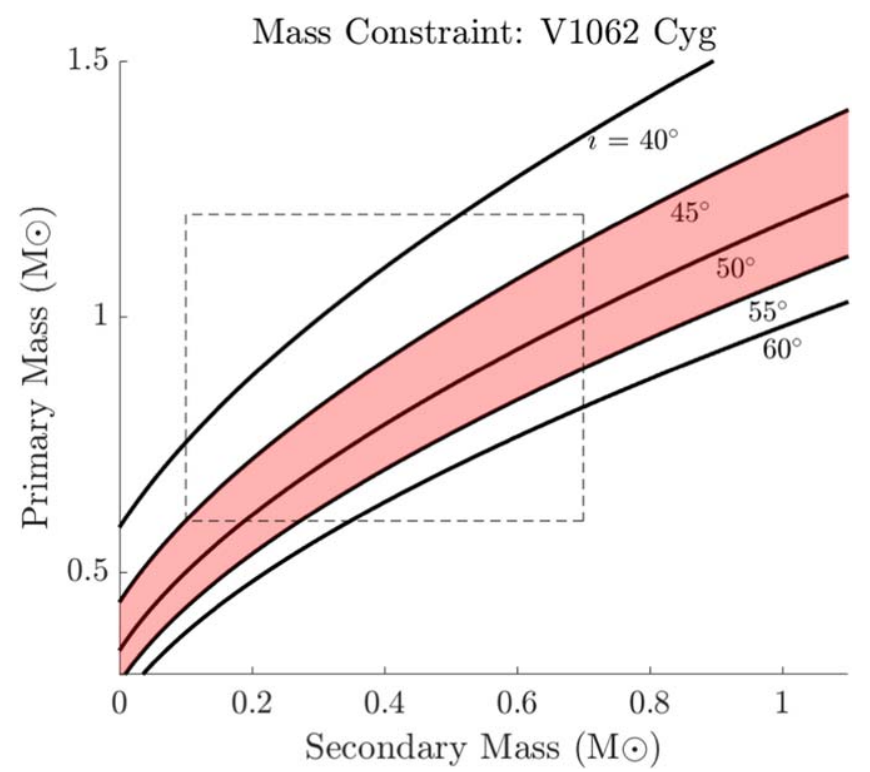

Figure 10. Mass constraints for V1062 Cyg. The shaded area indicates the inclination range we estimate from the light-curve model. The dotted box represents the reasonable range of masses for each component in the system.

again clearly accounts for most of the variation. ${ }^{8}$ Much of the data between phases 0.25 and 0.6 were affected by an intermittent telescope control problem that grossly degraded the images. The light-curve models give acceptable fits for $i=50^{\circ} \pm 5^{\circ}$; the curve drawn is for $i=50^{\circ}, M_{1}=0.8 M_{\odot}$, $M_{2}=0.5 M_{\odot}$, and a secondary star with $T_{\text {eff }}=3800 \mathrm{~K}$ and extra light equivalent to $3 \times 10^{-17} \mathrm{erg} \mathrm{cm}^{-2} \mathrm{~s}^{-1} \AA{ }^{-1}$. In Figure 10, we show the mass constraints for V1062 Cyg for a variety of potential inclinations.

\section{Discussion}

For ASASSN-14ho, we find the orbital period of $P=350.14 \pm 0.15$ minutes and determine the secondary's spectral type to be $\mathrm{K} 4 \pm 2$ subclasses. By modeling the light

\footnotetext{
8 These data were taken several nights after the spectra; when we computed phases using the absorption-velocity ephemeris, the observed ellipsoidal humps arrived earlier than expected, by $\sim 0.04$ cycle. Adjusting the period to a slightly shorter value, and the epoch $T_{0}$ to be a bit earlier, corrected this mismatch; the values used are given in the figure.
} 
curve, we estimate $i=52^{\circ} \pm 5^{\circ}$. The mass function and inclination suggest a white dwarf mass of $M_{1}>0.8 M_{\odot}$.

For V1062 Cyg, we find $P=348.25 \pm 0.60$ minutes, and classify the secondary as M0.5. The light curve yielded $i=50^{\circ} \pm 5^{\circ}$.

Both systems are DN with late-type secondary stars that dominate the optical flux. Both systems have orbital periods very close to $5.8 \mathrm{hr}$, well above the period gap of $2-3 \mathrm{hr}$ (Warner 1995). The period gap is conventionally understood to represent a transition between two mass accretion stages in the CV's lifetime (Howell et al. 2001).

Despite their nearly identical orbital periods, the two systems' secondary stars differ significantly in spectral type. Knigge et al. (2011) tabulate a semi-empirical sequence of the mean properties of $\mathrm{CV}$ secondaries, which gives $M_{2}=0.67 M_{\odot}$ and a spectral type of M0 for typical secondaries at this period. Our observations of V1062 Cyg are consistent with this, but the secondary of ASSASN-14ho is somewhat warmer and significantly less massive than their fiducial sequence. The empirical donor star data tabulated by Knigge (2006) does include secondaries as warm as that of ASASSN-14ho near this period.

The $\mathrm{H} \alpha$ line profile of V1062 Cyg shows an unusual narrow component that moves in phase with the secondary star, as well as a fainter diffuse component that moves approximately with the white dwarf and accretion disk. This may arise from irradiation of one face of the secondary star. Very narrow $\mathrm{H} \alpha$ lines phased with the secondary are sometimes seen in VY Scl stars during extreme low states (see, e.g., Weil et al. 2018 and references therein). Far-ultraviolet observations might clarify whether the white dwarf or accretion structures in this system are unusually hot.

This work uses data collected at the SAAO $1.0 \mathrm{~m}$ and $1.9 \mathrm{~m}$ and the MDM $1.3 \mathrm{~m}$ and $2.4 \mathrm{~m}$ telescopes. It is supported in part by the University of Cape Town and Dartmouth College's Frank J. Guarini Institute for International Education. We would also like to thank both Kathryn E. Weil and Meredith Joyce for their generous consultations. We thank the entire Dartmouth 2017 Astronomy Foreign Study Program: Carter H. Bartram, Abigail J. Buckley, Michael D. Cobb, Ana M. Colon, James G. Detweiler, John M. Elliot, Emily A. Golitzin, Alana M. Juric, Alexander S. Putter, and Anne M. Woronecki, for their unwavering support and assistance. This publication makes use of data products from the AAVSO Photometric All
Sky Survey (APASS), funded by the Robert Martin Ayers Sciences Fund and the National Science Foundation. The authors H.B., M.M., and P.W. acknowledge support through the National Research Foundation of South Africa; K.P. acknowledges funding by the National Research Foundation of South Africa (NRF) through a South African Radio Astronomy Observatory (SARAO) bursary, and University of Cape Town (UCT). We would like to thank Erek Alper for taking the photometric MDM observations of V1062 Cygni. Finally, we thank the anonymous referee for the careful reading and suggestions that significantly improved this paper.

\section{ORCID iDs}

Raphael E. Hviding (i) https://orcid.org/0000-0002-4684-9005 John R. Thorstensen (iD https://orcid.org/0000-00024964-4144

Kerry Paterson (1D https://orcid.org/0000-0001-8340-3486

\section{References}

Barwig, H., \& Schoembs, R. 1983, A\&A, 124, 287

Bochkarev, N. G., Karitskaia, E. A., \& Shakura, N. I. 1979, SvA, 23, 16

Claret, A., Hauschildt, P. H., \& Witte, S. 2012, A\&A, 546, A14

Coppejans, R., Gulbis, A. A. S., Kotze, M. M., et al. 2013, PASP, 125, 976

Crause, L. A., Carter, D., Daniels, A., et al. 2016, Proc. SPIE, 9908, 990827

Drake, A. J., Djorgovski, S. G., Mahabal, A., et al. 2009, ApJ, 696, 870

Gaia Collaboration, Brown, A. G. A., Vallenari, A., et al. 2018, A\&A, 616, A1

Gaia Collaboration, Prusti, T., de Bruijne, J. H. J., et al. 2016, A\&A, 595, A1

Henden, A. A., Levine, S., Terrell, D., \& Welch, D. L. 2015, AAS Meeting Abstracts, 225, 336.16

Hoffmeister, C. 1965, IBVS, 80, 1

Horne, K. 1986, PASP, 98, 609

Howell, S. B., Nelson, L. A., \& Rappaport, S. 2001, ApJ, 550, 897

Kepler, S. O., Kleinman, S. J., Nitta, A., et al. 2007, MNRAS, 375, 1315

Knigge, C. 2006, MNRAS, 373, 484

Knigge, C., Baraffe, I., \& Patterson, J. 2011, ApJS, 194, 28

North, R. C., Marsh, T. R., Kolb, U., Dhillon, V. S., \& Moran, C. K. J. 2002, MNRAS, 337, 1215

Prieto, J. L., Sturch, L., Madore, B., et al. 2014, ATel, 6619, 1

Ritter, H., \& Burkert, A. 1986, A\&A, 158, 161

Schneider, D. P., \& Young, P. 1980, ApJ, 238, 946

Schreiber, M. R., Zorotovic, M., \& Wijnen, T. P. G. 2016, MNRAS, 455, L16

Steeghs, D., Marsh, T., Knigge, C., et al. 2001, ApJL, 562, L145

Stetson, P. B. 1987, PASP, 99, 191

Thorstensen, J. R. 2013, PASP, 125, 506

Thorstensen, J. R., Alper, E. H., \& Weil, K. E. 2016, AJ, 152, 226

Thorstensen, J. R., \& Armstrong, E. 2005, AJ, 130, 759

Tonry, J., \& Davis, M. 1979, AJ, 84, 1511

Warner, B. 1995, CAS, 28, 1

Weil, K. E., Thorstensen, J. R., \& Haberl, F. 2018, AJ, 156, 231 